\title{
Land Surface Temperature Remote Sensing Inversion of Black Soil Area and Spatial Variation Analysis based on Landsat TM6
}

\author{
Guang-yu Zhang ${ }^{1,2}$, Wei Shen ${ }^{1}$, Zhao-zhuo Ouyang ${ }^{2}$, Fu-chao $\mathrm{Na}^{2}$, Fang \\ Yang $^{2}$ \\ ${ }^{1}$ School of Earth Sciences and Resources, China University of Geosciences, Beijing 100083, \\ China \\ ${ }^{2}$ Shenyang Institute of Geology and Mineral Resources, CGS, Shenyang 110034, China
}

Keywords: Temperature, Mono-window Algorithm, Inverse, Difference.

\begin{abstract}
Using Chagan Lake area as the study area and based on the DN value of sixth band of the Landsat 5 TM image of the region in 2011, this paper uses mono window algorithm to inverse the real surface temperature of the study area under the regional thermal radiation condition and curtained atmospheric transmissivity atmospheric transmission rate and emissivity. Then the inversion data and actual measured data are compared and analyzed. It is shown that the monowindow inversion algorithm achieved a high accuracy, and can better reflect the distribution of surface temperatures. On this basis, the temperature distribution of the region and its influencing factors are analyzed.
\end{abstract}

\section{Introduction}

Surface temperature is an important parameter for studying the physical processes of the earth's surface, an index to monitor the dynamic changes in the earth's resources and directly involved in the calculation of related models, such as "global circulation models", soil heat flow equation [1] etc.. The traditional surface temperature is obtained by multi-point observation, which lacks macroscopic. While thermal infrared remote sensing, with real-time and succession observation and other characteristics, is often used for surface temperature inversion.

At present, for different thermal infrared, there presents a variety of surface temperature inversion algorithms, mainly including atmospheric correction method, channel algorithm [2] and Mono-Window Algorithm. Channel algorithm was firstly used for calculating temperature of sea surface, then it was used for land surface after ameliorate. Though the traditional method of atmospheric correction (radiation conduction equations method) is feasible theoretically, the practical application has difficulties such as complicated calculation process and inaccurate and time-delay atmospheric profile data. Therefore, atmospheric simulation usually uses a standard atmospheric profile data to replace real-time measured data, or with a non-real-time data of atmospheric space exploration to replace real-time data, resulting in poor accuracy of surface temperature inversion when using atmospheric correction method (typically> $3{ }^{\circ} \mathrm{C}$ ) [3].

In 1996, Hurtado et al. [4] put forward a new method based atmospheric correction method for surface temperature inversion from TM data, based on the surface energy balance equation and standard atmospheric parameters. This method is very close to a new method of calculation. However, due to the calculation complexity and uncertainty of many parameters, such method is still hard to call a new algorithm. Later, Qin Zhihao [5-6] proposed a method of using TM6 data, according to the surface heat transfer equation to calculate a simple, feasible and high accuracy of surface temperature calculation method. In the absence of real-time atmospheric profiles data, Mono-Window Algorithm and single-channel algorithm can realize surface temperature inversion only based on near-surface air temperature, atmospheric moisture content and surface emissivity of these three parameters. Liu Sanchao has used this algorithm to study the thermal effects Zhangye City, Gansu Province [7]. TM6 Landsat remote sensing image data, because of its higher data definition, can be used to analyze thermal radiation and regional surface temperature difference. Therefore, in terms of accurate regional analysis, TM data is a better choice. 


\section{Research methods}

\section{The General Situation of Research Area}

Chagan Lake watershed and its adjacent land area are selected as the research area, in the range

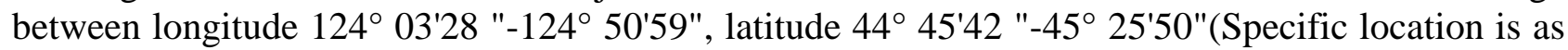
shown in Figure 1). Chagan Lake is located at the intersection area of the Nenjiang River and Huolinhe river in Qian Gorlos Mongolian Autonomous County, to south of the Songnen Plain, the northwest of Jilin Province. As the biggest inland lake in Jilin Province, Chagan Lake is about 37,000 hectares, with $\mathrm{pH}$ of about 8.3. The southwest area of Qianguo County is higher than northeast area. Its agrotype mainly includes chernozem, saline-alkali soil and meadow soil. It located in the warm temperate zone with mainland monsoon type climate and four distinctive seasons. The annual average sunshine there is 2879 hours, annual average temperature of $4.5^{\circ} \mathrm{C}$, annual effective accumulated temperature of $2916{ }^{\circ} \mathrm{C}$ and average annual precipitation of $450 \mathrm{~mm}$.

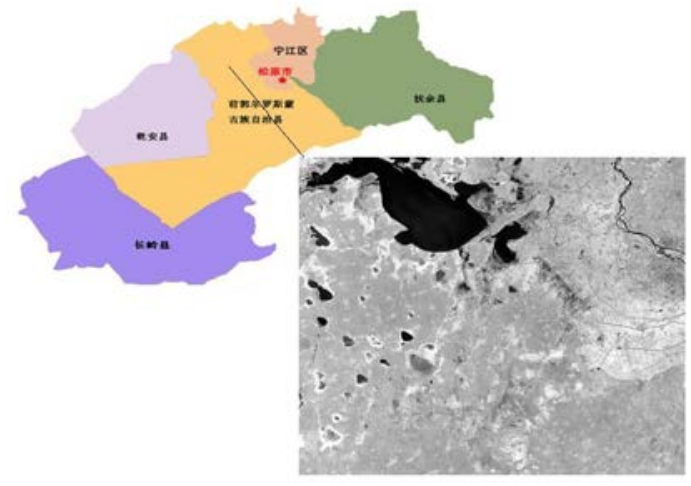

Fig.1: Qianguo County Study area

\section{Mono-Window Algorithm}

Radiative transfer equation is the basis for derivation of surface temperature inversion algorithm, and the principle Mono-Window Algorithm stems from the surface radiative transfer equation, which does not require a direct surface temperature inversion of atmospheric correction. Assuming a sunny day without significant vertical convection air, the atmosphere can be divided into several parallel air levels. If there lacks local real-time atmosphere profile data, the standard real-time atmospheric data and local meteorological data (mainly temperature and atmospheric moisture) can still be used in combination. The actual surface temperature can be estimated from the planet actual surface brightness temperature [8] only based on surface emissivity $\varepsilon$, the atmospheric equivalent temperature Ta and atmospheric transmittance $\tau$. The formula is as follows:

$$
\begin{array}{r}
T_{s}=\left\{a(1-C-D)+\left[(b-1)(1-C-D) \cdot T_{6}-D \cdot T_{a}\right]\right\} / C \\
C=\varepsilon \tau \\
D=(1-\tau)[1+(1-\varepsilon) \cdot \tau]
\end{array}
$$

In formula, $T_{s}$ refers to the surface temperature, $T_{6}$ is brightness temperature, and $T_{a}$ is the atmospheric equivalent temperature. $a$ and $b$ are coefficients (when the local surface temperature is $\left.0 \sim 70{ }^{\circ} \mathrm{C}, a=-67.355351, b=0.458606\right), C$ and $D$ is an intermediate variable, calculated as above, $\varepsilon$ is the surface emissivity, $\tau$ is the atmospheric transmittance. Before the temperature inversion, DN values need to be converted to radiance, gain and offset values use the value of USGS provided in 2003. The average atmospheric temperature is mainly estimated by method of Qin Zhihao, and brightness temperature can be calculated by Planck equation, or by using approximate formula the CAS provided for Earth Observation and Digital Earth Science Center.

\section{The certainty Surface emissivity}

This paper uses emissivity ratio estimation method proposed by Qin Zhihao to determine the surface emissivity of the study area within TM6 Band. Because the study area is mainly farmland and waters, so surface emissivity of the mixed pixel on natural surface in pixel scale can be calculated as follows: 


$$
\begin{gathered}
\varepsilon=P_{v} R_{v} \varepsilon_{v}+\left(1-P_{v}\right) \cdot R_{s} \varepsilon_{s}+d \varepsilon \\
R_{v}=0.9332+0.0585 P_{v} \\
R_{s}=0.9902+0.1068 P_{v} \\
P_{v}=\left(N D V I-N D V I_{s}\right) /\left(N D V I_{v}-N D V I_{s}\right)
\end{gathered}
$$

In above formula, $P_{v}$ is vegetation mixed pixel ratio, $R_{v}$ and $R_{s}$ are land surface emissivity of the vegetation and bare soil. Under the condition of relatively flat surface, we can value $d \varepsilon=0$; in the case of large difference of surface height, $d \varepsilon$ can simply estimate based on the proportions of vegetation. Since the study area flat terrain, a part of the Songnen Plains, so in this study, $d \varepsilon=0$. NDVI is the normalized difference vegetation index, $N D V I_{v}$ and $N D V I_{s}$ are $N D V I$ value of vegetation and bare soil. In general, if there is no obvious or completely vegetation on bare soil pixels or there is no detailed regional vegetation and soil spectra on map sheet, value $N D V I_{v}=0.70$ and $N D V I_{s}=0.05$ to estimate the approximate the vegetation coverage value[9].

\section{Data Processing}

By using the TM remote sensing image images at 14 o'clock on October 2, 2011 of research area, the ENVI software is used to for data geometric correction and projection transformation, and use of the band calculation function NEVI area matrix calculations.

\section{Analysis of the results}

\section{Result of Surface temperature inversion}

By using the Band Math function, the DNVI of the study area is as shown in Figure 2, and the land surface emissivity is as shown in Figure 3. Then through Mono-Window Algorithm, the land surface temperature chart of Chagan lake waters on 2 pm, Sep 2, 2011, is inverted as shown in Figure 4.

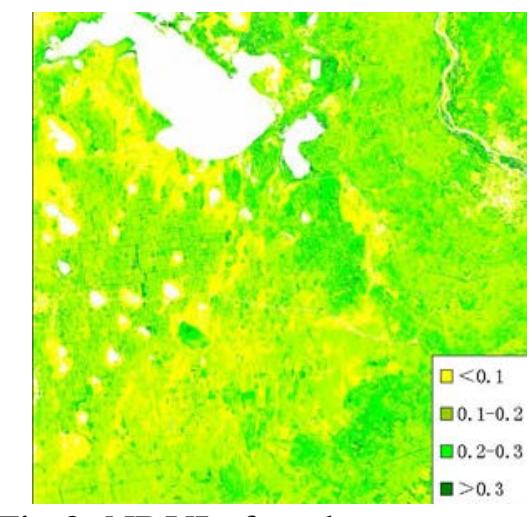

Fig.2: NDVI of study area

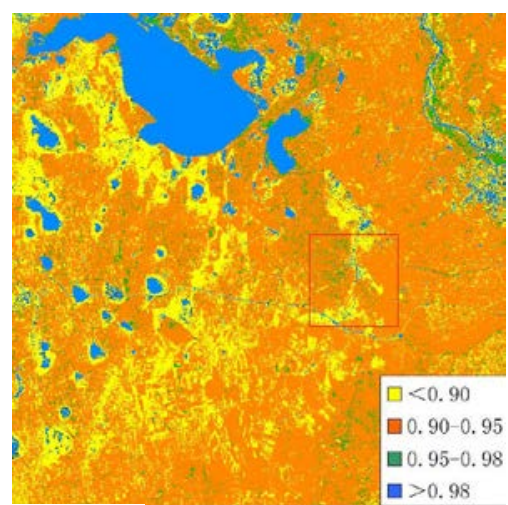

Fig.3: The land surface emissivity

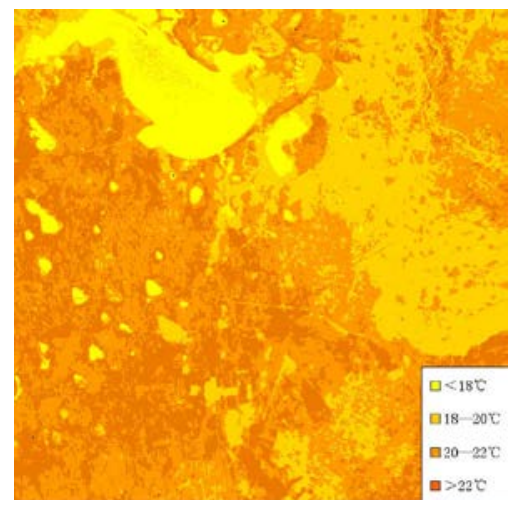

Fig.4: Surface temperature of study area 


\section{Error analysis}

In order to test the accuracy of the inversion results, the observation data and inversion results are compared and validated. The typical local ground objects are selected as the experimental points, including water, grassland, farmland, cities and towns, saline. The temperature of each ground object is sampled ten times to get the average temperature. Then we compared this average temperature with the average temperature of the $2 \times 2$ pixel corresponding position on the graph according to the specific location of the actual measurement data. Table 1 shows the land surface temperature data of experimental points inverted by TM6 data, and the measured temperature and inversion temperature is compared and error analyzed.

Table 1: Error analysis of surface temperature

\begin{tabular}{ccccc}
\hline Longitude and latitude & $\begin{array}{c}\text { Object } \\
\text { types }\end{array}$ & $\begin{array}{c}\text { Measured } \\
\text { temperature }\end{array}$ & $\begin{array}{c}\text { Inversion } \\
\text { temperature }\end{array}$ & Error \\
\hline $124^{\circ} 21^{\prime} 24^{\prime \prime} \mathrm{N} / 45^{\circ} 15^{\prime} 00^{\prime \prime} \mathrm{E}$ & Waters & 15.13 & 17.26 & -2.13 \\
$124^{\circ} 21^{\prime} 44^{\prime \prime} \mathrm{N} / 45^{\circ} 14^{\prime} 23^{\prime \prime} \mathrm{E}$ & Meadow & 16.95 & 19.33 & -2.38 \\
$124^{\circ} 41^{\prime} 32^{\prime \prime} \mathrm{N} / 45^{\circ} 12^{\prime} 33^{\prime \prime} \mathrm{E}$ & Farmland & 17.63 & 20.03 & -2.4 \\
$124^{\circ} 45^{\prime} 34^{\prime \prime} \mathrm{N} / 44^{\circ} 50^{\prime} 53^{\prime \prime} \mathrm{E}$ & Town & 18.79 & 22.23 & -3.44 \\
$124^{\circ} 01^{\prime} 24^{\prime \prime} \mathrm{N} / 45^{\circ} 13^{\prime} 22^{\prime \prime} \mathrm{E}$ & Saline- & 19.02 & 22.57 & -3.55 \\
& & & &
\end{tabular}

\section{The Analysis of Land Surface temperatures Differences}

According to inversion date gained by Mono-Window Algorithm, the temperature date differs significantly between land surface and waters, so does temperature dates among different land surfaces. Heat capacity of land surface is smaller than waters under the sunlight, therefore under the same sun heat, the temperature of land will climbs quicker than water, which is the reason why the temperature of land surface is higher than waters [10].

According to temperature inversion results, the temperature differences among land surfaces are also obvious. By contrast with the data of measured temperature, it is found that the area with higher temperature is basically a residential area or Saline area, the lower area is basically grassland and farmland. Various temperatures are decided by different ground objects covered on land, which shows up different physical and chemical characteristics. The reflectance infrared band in the saline-alkali land and building area is lower than the grassland or farmland.

There are two reasons why the surface temperature of Farmland is $0.68{ }^{\circ} \mathrm{C}$ higher than grassland averagely as shown in table one. Firstly, farmland and grassland have different vegetational type and coverage, resulting in the moisture of farmland evaporated faster than grassland, i.e., soil moisture content of the grassland is higher than its adjacent farmland [11-12]. Therefore when they receive the same heat, the temperature of the farmland rise higher than the grassland; Secondly, long-term cultivation of the farmland has changed the microflora in soil , and the number of fungi, actinomycetes, bacteria and other microorganisms on soil surface are bigger than grassland ecosystem. The heat released by microbial activity also helps improve the soil temperature.

Temperature of cities and towns is higher than the urban surrounding environment, the so-called heat island effect. It was firstly affected by the characteristic of urban underlying surface. The thermodynamic properties of urban underlying surface are changed by numerous artificial structures, such as concrete buildings, asphalt, various buildings walls, etc., leading to smaller reflectance of urban area and accelerating heat conduction speed. With the features of faster heat reception, smaller specific heat capacity, these artificial structures receive more heat, heat up faster, consume less heat while evaporating, and dissipate less heat than natural underlying surface under the same conditions of thermal radiation. Therefore, it has higher surface temperature than natural underlying surface. The temperature is also affected by artificial heat source, such as heat daily emitted by manufacturing, transportation, and other fuels (coal, natural gas, etc.) used in human daily life.

The spectral characteristics of soil salinization is affected by soil characteristics, such as mineral content, water content, organic content, soil structure, roughness and degree of weathering, vegetation coverage, etc. [13], resulting in the reflection characteristics of soil salinization land surface manifestations-related. The study of Mougenot et al [14] shows that different saline-alkali 
soil has different salt content and thickness, gassy surface consisting of Granhle soil and crystalline salt, and gassy surface layer. It also indicates that different surface configuration has different surface roughness and presents different reflectance signature. Generally, the saline-alkali soil has greater reflectance in the visible light and near-infrared band than other earth surface. the reflection in the thermal infrared band is weaker, causing its temperature higher than the surrounding ambient temperature.

\section{Conclusion}

This paper, by using Mono-Window Algorithm, conducts surface temperature inversion to study are and compares inversion results and the measured temperature of the study area. It is shown that : (1) it is very close in overall temperature trend of temperature inversion data and actual measured data, however, , the inversion data achieved by Mono-Window Algorithm is higher compared with the measured data, with a difference of about $2.78{ }^{\circ} \mathrm{C}$; (2) among five typical ground cover types , compared with the measured data, temperature inversion data of waters, grassland and farmland are higher by2.13 ${ }^{\circ} \mathrm{C}, 2.38{ }^{\circ} \mathrm{C}$ and $2.4{ }^{\circ} \mathrm{C}$ respectively. Cities and saline-alkali soil obviously have greater data difference of $3.44{ }^{\circ} \mathrm{C}$ and $3.55{ }^{\circ} \mathrm{C}$, respectively. These data differences presents an incremental trend of waters $\rightarrow$ natural surfaces $\rightarrow$ cities, indicating the higher temperature distribute area of the city has the greater temperature errors.

This paper hopes the research of surface temperature inversion and temperature difference could play a helpful role in agriculture and ecology development of the study zone by the comparison and analysis of the measured and inverted temperature data and their spatial difference. Though the surface temperature difference of dry land is mainly determined by vegetation, the experimental data is still effective because the image used in this paper is captured in October.

There are three key parameters in using Mono-Window Algorithm to study the spatial difference of surface temperature, which are atmospheric transmittance, the average atmospheric temperature and surface emissivity. This paper mainly focus the study on the average atmospheric temperature, How estimation errors of atmospheric transmittance and surface emissivity affect inversion data remains to be further studied discussed and There are still some shortcomings, such as the surface temperature inversion accuracy need to be improved.

\section{Acknowledgements}

The research work was supported by China Geological Survey Project under Grant No. 1212011220845.

\section{References}

[1] Zhao Ying-shi. Application of the principles and methods of remote sensing, Beijing: Science Press, 2003.

[2] Chen Zi-tong. Study on SST With GMS Observation and The Method of Analysis Technique. Journal of Tropical Meteorology 17(3), pp. 235-241, 2001.

[3] Luo Zhi-yong, Liu Han-hu, Yang Wu-nian. The Application Study of Mono-window Algorithm in Chengdu for Land Temperature Retrieval. Journal of Tropical Meteorology, 23(4), pp. 409-412, 2007.

[4] Hur tado E, Vidal A, Caselles V. Comparison of two atmospheric correction methods for Landsat TM thermal band. International Journal of Remote Sensing, 17(6), pp. 237-247, 1996.

[5] Qin Zhi-hao, Zhang Ming-hua, Arnon Karnieli,et al. Mono-window Algorithm for Retrieving Land Surface Temperature from Landsat TM6 data. Acta Geographica Sinica, 56 (4) pp. 456466, 2001.

[6] Qin Zhi-hao, Li Wen-juan, Zhang Ming-hua. Esimating of The Essential Atmospheric Parmeters of Mono-window Algorithm for Surface Temperature Retrieval From Landsat TM6. Remote Sensing For Land \& Resources, 56 (2), pp. 37-43, 2003. 
[7] Liu San-chao, Zhang Wan-chang. A Remote Sensing Study of The Urban Thermal Effect on Zhangye and Its Surrounding Oasis Area. Remote Sensing For Land \& Resources, 23(4), pp. 17-21, 2003.

[8] QIN Zhi-hao ,Karnielia, Berliner p. A mono-window algorithm for retrieval land surface temperature from Landsat TM data and its application to the Israel-Egypt border region. Inter $J$ of Remote Sensing, 22(18) pp. 3719-3746, 2001.

[9] Ye Zhi-wei,Qin Zhi-hao,Gong Hui-li. Land Surface Temperature Retrieval and Spatial Variation Analysis Using Landsat TM6 in Hongze Lake. Journal of Capital Normal University(Natural Science Edition), 30(1) pp.88-95, 2009 .

[10]Yang Dayuan. Physical Geography.Nanjing: Nanjing University Press, 2001.

[11]Guo Rui-ping, Mo Xing-guo. Differences in forest, grassland and farmland evapotranspiration. Chinese Journal of Applied Ecology, 18(8) , pp. 1751-1757, 2007.

[12]Qin Hong-ling, Gao Wang-sheng, Li Chun-yang. Impacts of no-tillage on soil temperature of field in Ecotone of North China. Transactions of the Chinese Society of Agricultural Engineering, 23(1), pp. 40-47, 2007.

[13]Wu Yun-zhao, Tian Qing-jiu, Ji Jun-feng. Soil Remote Sensing Research Theory Method and Application. Remote Sensing Information, 5(1) , pp. 40-47. 2003.

[14]Mougenot B, Pouget M, Epema G. Remote Sensing of Salt2 affected Soils. Remot Sensing Reviews, 7(2) , pp. 241-259, 1993. 